commissioner of fisheries, Spencer Fullerton Baird. Edwin Linton

WAShington AND JeFFerson College

\section{OBSERVATIONS ON THE SOLAR ECLIPSE MADE BY THE CROCKER EXPEDITION OF THE LICK OB- SERVATORY 1}

THE preparations for observing the total eclipse, including the standardizing of the photographic plates by means of a standard lamp and the loading of the plate holders were completed Friday evening. The weather conditions were not promising Saturday morning, with the sky completely covered with clouds of medium thickness, and these continued throughout the day, except for a short break, which proved to be one of the most remarkable coincidences known to me.

The prospects for a clear sky were apparently hopeless during the long hours of waiting, almost up to the time of totality. Fifty minutes before the moon's shadow was due to reach us we noticed a thinning of the clouds near the western horizon. It seemed hopeless to expect that the rift would continue or reach the region of the sky that we were interested in, bat it did.

A very small area of the blue sky free from clouds had the sun at its center exactly at the center of the total phase, and all other parts of the sky were clouded. This region cleared not more than a minute before the beginning of totality, and clouds again covered the sun less than a minute after the passing of the shadow.

All of the instruments and all of the observers were ready, and the program went through as planned. Goldendale is situated exactly on the central line of the eclipse path. Observations made by my colleague, Professor Tucker, at Mount Hamilton, several months ago, had shown that the moon was slightly ahead of its predicted place, and he estimated that the eclipse would occur twenty seconds earlier than the time set down' for it in the

1 Press despatch revised by the author for publication in ScIENCE.
Nautical Almanac. We accordingly allowed for this in our program, and totality began two seconds later than Tucker's predicted time. The observed duration, one minute and fiftyseven seconds, agreed perfectly with the Almanac data.

\section{DARKNESS UNUSUALLY PRONOUNCED.}

The eclipse was a very dark one, the darkest of the six observed by me. The reading of newspaper print would have been difficult under the open sky. The chickens retired as if for the night. They were heard to give the morning cock crows before emerging a few minutes later. It was probably the shortest night in all their lives.

The eclipse phenomena, both celestial and terrestrial, formed a spectacle indescribably unusual and magnificent. The solar corona was, of course, the center of interest. It seemed brighter than usual, and its general outline was more elongated than we had expected, in view of the fact that we are not far from sunspot maximum.

The coronal streamers were visible two and one half solar diameters to the east and west of the sun, but scarcely more than one diameter to the north and south, and the outline form was approximately triangular, with the eastern steamers converging to a sharp vertex at their most easterly point and the western streamers diverging to the base of the triangle at the most westerly points. The photographs thus far developed confirm the naked-eye description and extend the east and west streamers out to more than three diameters.

The solar prominences were numerous and large, as we should expect at a time of great sun-spot activity, but these did not concern us greatly, as they can be observed well without an eclipse. However, the prominences contribute greatly to the interest of the photographs, as the arching of the coronal streamers around the prominences is conspicuous, leaving no doubt that the forces which produce the prominences are controlling the forms of coronal streamers in their neighborhoods.

A few of the twenty-six photographs, secured 
with cameras of focal lengths from eleven inches up to forty feet, have been developed, and the details of coronal structure were recorded with admirable sharpness, showing that the instruments were in good adjustment, and, what is equally important, that the earth's atmosphere traversed by the coronal radiations was in a tranquil state.

TEST OF THE EINSTEIN THEORY.

Four cameras of fifteen feet focus, using plates of 14 by 17 inches, recorded the brighter stars existing in the region immediately surrounding the sun, though vastly further away, with apparent success, but their examination and suitable study can not occur until many days after the plates reach home. It is hoped that the measured positions of the recorded stars will serve as a test of correctness or falsity of the so-called Einstein theory of relativity, a subject which has occupied a foremost position in the speculations of physicists and others during the last decade.

If Einstein's hypothesis represents the truth, then the positions of the stars on the plates should be affected during the action of the sun's gravitations upon the rays proceeding from the stars while the rays are passing closely by the sun on their way to the earth. The test as an eclipse problem has never been made before, and it may be the only satisfactory test known to physicists, but whether our work will contribute evidence of value remains to be seen.

The same four plates should contribute something of value as to the existence or nonexistence of any known bodies, such as the hypothetic planet Vulcan, in the vicinity of the sun. We expect to find no strange objects in the region commanded by the plates, and that the evidence will be wholly negative, but such comments have no weight a present.

Two spectographs were successful in their purpose of recording images of the stratum of green coronium gas enveloping the sun. This stratum is relatively thick over the east and west areas of the solar surface, but thin over the polar regions. The distribution of coronium is very irregular. The accurate position of the green coronium line can be determined with good accuracy from the images obtained with a three-prism spectrograph.

The general spectrum was recorded in good strength with two spectrographs. The evidences of polarized light are strong and definite on photographs obtained with two polarigraphs. Just what is the nature of the evidence which they will afford as to the composition of the coronal streamers will depend upon quantitative measures demanding time and discussion.

Four photographs were obtained by Miss Glancy, assistant in the National Observatory of the Argentine Republic, for that institution, to serve as a basis for determining the total quantity of light radiated by the coronal structure. She has taken the plates with her for later development and study. It is possible that her results will be affected unfavorably by the clouds which extended close up to the coronal structure, and it is possible that some of our Einstein and Vulcan plates are similarly damaged.

We constantly revert in thought and speech to the remarkable coincidence which brought a very small rift in the clouds, the only rift visible in the entire sky centrally over the sun and corona during the three minutes-and the three minutes alone-which interested us.

The expedition was composed of Director and Mrs. Campbell, Astronomer H. D. Curtis, Assistant Astronomer and Mrs. J. E. Moore, and Foreman J. E. Hoover, of Lick Observatory; Professor E. P. Lewis, of the Department of Physics, University of California; Mr. A. H. Babcock, consulting electrical engineer of the Southern Pacific Co., San Francisco; Miss Leah B. Allen, instructor in Wellesley College; Miss Estelle Glancy, of the National Observatory at Cordoba, Argentina; Professor S. L. Boothroyd, University of Washington; Dr. Ambrose Swasey, of the Warner \& Swasey Co., Cleveland; Dr. John A. Brashear, Allegheny, Pennsylvania; Professor Douglas Campbell, Stanford University; Dr. J. S. Plaskett, director of the Dominion Observatory, Victoria, B. C. W. W. CAMPBelL

LICK OBSERTATORY 